\title{
Generalized degenerate Bernoulli numbers and polynomials arising from Gauss hypergeometric function
}

\author{
Taekyun Kim , Dae San Kim², Lee-Chae Jang ${ }^{3 *}$, Hyunseok Lee ${ }^{1}$ and Hanyoung Kim ${ }^{1}$
}

"Correspondence:

Lcjang@konkuk.ac.kr

${ }^{3}$ Graduate School of Education,

Konkuk University, Seoul, 143-701, Republic of Korea

Full list of author information is

available at the end of the article

\section{Springer}

\begin{abstract}
A new family of $p$-Bernoulli numbers and polynomials was introduced by Rahmani (J. Number Theory 157:350-366, 2015) with the help of the Gauss hypergeometric function. Motivated by that paper and in the light of the recent interests in finding degenerate versions, we construct the generalized degenerate Bernoulli numbers and polynomials by means of the Gauss hypergeometric function. In addition, we construct the degenerate type Eulerian numbers as a degenerate version of Eulerian numbers. For the generalized degenerate Bernoulli numbers, we express them in terms of the degenerate Stirling numbers of the second kind, of the degenerate type Eulerian numbers, of the degenerate $p$-Stirling numbers of the second kind and of an integral on the unit interval. As to the generalized degenerate Bernoulli polynomials, we represent them in terms of the degenerate Stirling polynomials of the second kind.
\end{abstract}

MSC: 11B68; 11B73; 11B83; 33C05

Keywords: Generalized degenerate Bernoulli numbers; Generalized degenerate Bernoulli polynomials; Degenerate type Eulerian numbers

\section{Introduction}

We have witnessed in recent years that many interesting arithmetic and combinatorial results were obtained in studying degenerate versions of some special polynomials and numbers (see [7-13] and the references therein), which was initiated by Carlitz when he introduced the degenerate Stirling, Bernoulli and Euler numbers in [3]. The studies have been done with various different tools such as combinatorial methods, generating functions, umbral calculus, $p$-adic analysis, differential equations, special functions, probability theory and analytic number theory. It should be noted that studying degenerate versions can be done not only for polynomials but also for transcendental functions. Indeed, the degenerate gamma functions were introduced as a degenerate version of ordinary gamma functions in [9]. The degenerate special polynomials and numbers have potential to find diverse applications in many areas just as 'ordinary' special polynomials and numbers play very important role in science and engineering as well as in mathematics. Indeed, it was

(c) The Author(s) 2021. This article is licensed under a Creative Commons Attribution 4.0 International License, which permits use, sharing, adaptation, distribution and reproduction in any medium or format, as long as you give appropriate credit to the original author(s) and the source, provide a link to the Creative Commons licence, and indicate if changes were made. The images or other third party material in this article are included in the article's Creative Commons licence, unless indicated otherwise in a credit line to the material. If material is not included in the article's Creative Commons licence and your intended use is not permitted by statutory regulation or exceeds the permitted use, you will need to obtain permission directly from the copyright holder. To view a copy of this licence, visit http://creativecommons.org/licenses/by/4.0/ 
shown in $[10,11]$ that the expressions of the probability distributions of appropriate random variables can be represented in terms of both the degenerate $\lambda$-Stirling polynomials of the second kind and the $r$-truncated degenerate $\lambda$-Stirling polynomials of the second kind.

In [14], Rahmani introduced a new family of $p$-Bernoulli numbers and polynomials by means of the Gauss hypergeometric function which reduce to the classical Bernoulli numbers and polynomials for $p=0$. Motivated by that paper and as a degenerate version of those numbers and polynomials, in this paper we introduce the generalized degenerate Bernoulli numbers and polynomials again in terms of the Gauss hypergeometric function which reduce to the Carlitz degenerate Bernoulli numbers and polynomials for $p=0$. In addition, we introduce the degenerate type Eulerian numbers as a degenerate version of Eulerian numbers. The aim of this paper is to study the generalized degenerate Bernoulli numbers and polynomials and to show their connections to other special numbers and polynomials. Among other things, for the generalized degenerate Bernoulli numbers we express them in terms of the degenerate Stirling numbers of the second kind, of the degenerate type Eulerian numbers, of the degenerate $p$-Stirling numbers of the second kind and of an integral on the unit interval. As to the generalized degenerate Bernoulli polynomials, we represent them in terms of the degenerate Stirling polynomials of the second kind. For the rest of this section, we recall the necessary facts that are needed throughout this paper.

For any $\lambda \in \mathbb{R}$, the degenerate exponential functions are defined by

$$
e_{\lambda}^{x}(t)=\sum_{n=0}^{\infty}(x)_{n, \lambda} \frac{t^{n}}{n !}, e_{\lambda}(t)=e_{\lambda}^{1}(t) \quad(\text { see }[6,9])
$$

where $(x)_{0, \lambda}=1,(x)_{n, \lambda}=x(x-\lambda) \cdots(x-(n-1) \lambda)(n \geq 1)$. Note that $\lim _{\lambda \rightarrow 0} e_{\lambda}^{x}(t)=e^{x t}$.

Let $\log _{\lambda}(t)$ be the compositional inverse function of $e_{\lambda}(t)$ with $\log _{\lambda}\left(e_{\lambda}(t)\right)=e_{\lambda}\left(\log _{\lambda}(t)\right)=$

$t$. Then we have

$$
\log _{\lambda}(1+t)=\sum_{n=1}^{\infty} \lambda^{n-1}(1)_{n, 1 / \lambda} \frac{t^{n}}{n !} \quad(\text { see }[7])
$$

In [7], the degenerate Stirling numbers of the first kind are defined by

$$
(x)_{n}=\sum_{l=0}^{n} S_{1, \lambda}(n, l)(x)_{l, \lambda} \quad(n \geq 0),
$$

where $(x)_{0}=1,(x)_{n}=x(x-1)(x-2) \cdots(x-n+1)(n \geq 1)$.

As the inversion formula of (3), the degenerate Stirling numbers of the second kind are defined by

$$
(x)_{n, \lambda}=\sum_{k=0}^{n} S_{2, \lambda}(n, k)(x)_{k} \quad(n \geq 0)(\text { see }[7])
$$

From (3) and (4), we note that

$$
\frac{1}{k !}\left(\log _{\lambda}(1+t)\right)^{k}=\sum_{n=k}^{\infty} S_{1, \lambda}(n, k) \frac{t^{n}}{n !}
$$


and

$$
\frac{1}{k !}\left(e_{\lambda}(t)-1\right)^{k}=\sum_{n=k}^{\infty} S_{2, \lambda}(n, k) \frac{t^{n}}{n !} \quad(k \geq 0)(\text { see [7]). }
$$

It is well known that the Gauss hypergeometric function is given by

$$
{ }_{2} F_{1}\left(\begin{array}{c}
a, b \\
c
\end{array} \mid x\right)=\sum_{k=0}^{\infty} \frac{\langle a\rangle_{k}\langle b\rangle_{k}}{\langle c\rangle_{k}} \frac{x^{k}}{k !} \quad(\text { see }[1,2,12])
$$

where $\langle a\rangle_{0}=1,\langle a\rangle_{k}=a(a+1) \cdots(a+k-1),(k \geq 1)$.

The Euler transformation formula is given by

$$
{ }_{2} F_{1}\left(\begin{array}{c}
a, b \\
c
\end{array} \mid x\right)=(1-x)^{c-a-b}{ }_{2} F_{1}\left(\begin{array}{c}
c-a, c-b \\
c
\end{array} \mid x\right) \quad(\text { see }[1,2]) .
$$

The Eulerian number $\left(\begin{array}{l}n \\ k\end{array}\right)$ is the number of permutation $\{1,2,3, \ldots, n\}$ having $k$ permutation ascents. The Eulerian numbers are given explicitly by the finite sum

$$
\left\langle\begin{array}{l}
n \\
k
\end{array}\right\rangle=\sum_{j=0}^{k+1}(-1)^{j}\left(\begin{array}{c}
n+1 \\
j
\end{array}\right)(k-j+1)^{n} \quad(n, k \geq 0, n \geq k)
$$

and

$$
\sum_{k=0}^{n}\left\langle\begin{array}{l}
n \\
k
\end{array}\right\rangle=n ! \quad(\text { see }[4,5])
$$

For $n, m \geq 0$, we have

$$
\left\langle\begin{array}{l}
n \\
m
\end{array}\right\rangle=\sum_{k=0}^{n-m} S_{2}(n, k)\left(\begin{array}{c}
n-k \\
m
\end{array}\right)(-1)^{n-k-m} k ! \quad(\text { see }[5])
$$

and

$$
x^{n}=\sum_{k=0}^{n}\left\langle\begin{array}{l}
n \\
k
\end{array}\right\rangle\left(\begin{array}{c}
x+k \\
n
\end{array}\right) \quad(\text { see }[4,5])
$$

Recently, the degenerate Stirling polynomials of the second kind were defined by

$$
\frac{1}{k !}\left(e_{\lambda}(t)-1\right)^{k} e_{\lambda}^{x}(t)=\sum_{n=k}^{\infty} S_{2, \lambda}(n, k \mid x) \frac{t^{n}}{n !}(k \geq 0) \quad(\text { see [8]). }
$$

Thus, by (13), we get

$$
S_{2, \lambda}(n, k \mid x)=\sum_{l=k}^{n}\left(\begin{array}{l}
n \\
l
\end{array}\right) S_{2, \lambda}(l, k)(x)_{n-l, \lambda} \quad(\text { see }[8])
$$




$$
=\sum_{l=0}^{n}\left(\begin{array}{l}
n \\
l
\end{array}\right) S_{2, \lambda}(l, k)(x)_{n-l, \lambda} \quad(n \geq 0) .
$$

For $x=0, S_{2, \lambda}(n, k)=S_{2, \lambda}(n, k \mid 0)(n, k \geq 0, n \geq k)$, are called the degenerate Stirling numbers of the second kind.

Carlitz introduced the degenerate Bernoulli polynomials given by

$$
\frac{t}{e_{\lambda}(t)-1} e_{\lambda}^{x}(t)=\sum_{n=0}^{\infty} \beta_{n, \lambda}(x) \frac{t^{n}}{n !} \quad(\text { see [3]). }
$$

When $x=0, \beta_{n, \lambda}=\beta_{n, \lambda}(0)(n \geq 0)$, are called the degenerate Bernolli numbers.

\section{Generalized degenerate Bernoulli numbers}

By (1) and (2), we get

$$
\begin{aligned}
\frac{t}{e_{\lambda}(t)-1} & =\frac{1}{e_{\lambda}(t)-1} \sum_{n=1}^{\infty} \lambda^{n-1}(1)_{n, 1 / \lambda} \frac{1}{n !}\left(e_{\lambda}(t)-1\right)^{n} \\
& =\sum_{k=0}^{\infty} \frac{\lambda^{k}(1)_{k+1,1 / \lambda}}{k+1} \cdot \frac{1}{k !}\left(e_{\lambda}(t)-1\right)^{k} \\
& =\sum_{k=0}^{\infty} \frac{\lambda^{k}(1)_{k+1,1 / \lambda}}{k+1} \sum_{n=k}^{\infty} S_{2, \lambda}(n, k) \frac{t^{n}}{n !} \\
& =\sum_{n=0}^{\infty}\left(\sum_{k=0}^{n} \frac{\lambda^{k}(1)_{k+1,1 / \lambda}}{k+1} S_{2, \lambda}(n, k)\right) \frac{t^{n}}{n !} .
\end{aligned}
$$

Therefore, by (15) and (16), we obtain the following theorem.

Theorem 1 For $n \geq 0$, we have

$$
\beta_{n, \lambda}=\sum_{k=0}^{n} \frac{\lambda^{k}(1)_{k+1,1 / \lambda}}{k+1} S_{2, \lambda}(n, k) .
$$

Replacing $t$ by $\log _{\lambda}(1+t)$ in (15), we get

$$
\begin{aligned}
\frac{\log _{\lambda}(1+t)}{e_{\lambda}\left(\log _{\lambda}(1+t)\right)-1} & =\sum_{k=0}^{\infty} \beta_{k, \lambda} \frac{1}{k !}\left(\log _{\lambda}(1+t)\right)^{k} \\
& =\sum_{k=0}^{\infty} \beta_{k, \lambda} \sum_{n=k}^{\infty} S_{1, \lambda}(n, k) \frac{t^{n}}{n !} \\
& =\sum_{n=0}^{\infty}\left(\sum_{k=0}^{n} S_{1, \lambda}(n, k) \beta_{k, \lambda}\right) \frac{t^{n}}{n !} .
\end{aligned}
$$

On the other hand, by (2), we get

$$
\frac{\log _{\lambda}(1+t)}{e_{\lambda}\left(\log _{\lambda}(1+t)\right)-1}=\frac{1}{t} \log _{\lambda}(1+t)=\frac{1}{t} \sum_{n=1}^{\infty} \lambda^{n-1}(1)_{n, 1 / \lambda} \frac{t^{n}}{n !}
$$




$$
=\sum_{n=0}^{\infty} \frac{\lambda^{n}(1)_{n+1,1 / \lambda}}{n+1} \frac{t^{n}}{n !}
$$

Therefore, by (17) and (18), we obtain the following theorem.

Theorem 2 For $n \geq 0$, we have

$$
\sum_{k=0}^{n} S_{1, \lambda}(n, k) \beta_{k, \lambda}=\frac{1}{n+1} \lambda^{n}(1)_{n+1,1 / \lambda}
$$

From (15) and (16), we note that

$$
\begin{aligned}
\sum_{n=0}^{\infty} \beta_{n, \lambda} \frac{t^{n}}{n !} & =\frac{1}{e_{\lambda}(t)-1} \sum_{n=1}^{\infty} \lambda^{n-1}(1)_{n, 1 / \lambda} \frac{1}{n !}\left(e_{\lambda}(t)-1\right)^{n} \\
& =\sum_{n=0}^{\infty} \frac{(-1)^{n}(1)_{n+1,1 / \lambda} \lambda^{n} n !}{(n+1) !} \frac{\left(1-e_{\lambda}(t)\right)^{n}}{n !} \\
& =\sum_{n=0}^{\infty} \frac{\langle 1-\lambda\rangle_{n}\langle 1\rangle_{n}}{\langle 2\rangle_{n}} \frac{\left(1-e_{\lambda}(t)\right)^{n}}{n !} \\
& ={ }_{2} F_{1}\left(\begin{array}{c}
1-\lambda, 1 \\
2
\end{array} \mid 1-e_{\lambda}(t)\right) .
\end{aligned}
$$

In view of (19), we may consider the generalized degenerate Bernoulli numbers given in terms of Gauss hypergeometric function by

$$
{ }_{2} F_{1}\left(\begin{array}{c}
1-\lambda, 1 \\
p+2
\end{array} \mid 1-e_{\lambda}(t)\right)=\sum_{n=0}^{\infty} \beta_{n, \lambda}^{(p)} \frac{t^{n}}{n !}
$$

where $p \in \mathbb{Z}$ with $p \geq-1$. When $p=0, \beta_{n, \lambda}^{(0)}=\beta_{n, \lambda},(n \geq 0)$.

Let us take $p=-1$ in (20). Then we have

$$
\begin{aligned}
\sum_{n=0}^{\infty} \beta_{n, \lambda}^{(-1)} \frac{t^{n}}{n !} & ={ }_{2} F_{1}\left(\begin{array}{c}
1-\lambda, 1 \\
1
\end{array} \mid 1-e_{\lambda}(t)\right) \\
& =\sum_{n=0}^{\infty} \frac{(\lambda-1)_{n}}{n !}\left(e_{\lambda}(t)-1\right)^{n}=\sum_{n=0}^{\infty}\left(\begin{array}{c}
\lambda-1 \\
n
\end{array}\right)\left(e_{\lambda}(t)-1\right)^{n} \\
& =e_{\lambda}^{\lambda-1}(t)=\sum_{n=0}^{\infty}(\lambda-1)_{n, \lambda} \frac{t^{n}}{n !} .
\end{aligned}
$$

By comparing the coefficients on both sides of (21), we get

$$
\beta_{n, \lambda}^{(-1)}=(\lambda-1)_{n, \lambda} \quad(n \geq 0) .
$$

From (20), we note that

$$
\sum_{n=0}^{\infty} \beta_{n, \lambda}^{(p)} \frac{t^{n}}{n !}={ }_{2} F_{1}\left(\begin{array}{c}
1-\lambda, 1 \\
p+2
\end{array} \mid 1-e_{\lambda}(t)\right)=\sum_{k=0}^{\infty} \frac{\langle 1-\lambda\rangle_{k}\langle 1\rangle_{k}}{\langle p+2\rangle_{k}} \frac{\left(1-e_{\lambda}(t)\right)^{k}}{k !}
$$




$$
\begin{aligned}
& =(p+1) ! \sum_{k=0}^{\infty} \frac{\lambda^{k}(1)_{k+1,1 / \lambda} k !}{(p+k+1) !} \frac{1}{k !}\left(e_{\lambda}(t)-1\right)^{k} \\
& =(p+1) ! \sum_{k=0}^{\infty} \frac{\lambda^{k}(1)_{k+1,1 / \lambda} k !}{(p+k+1) !} \sum_{n=k}^{\infty} S_{2, \lambda}(n, k) \frac{t^{n}}{n !} \\
& =\sum_{n=0}^{\infty}\left(\sum_{k=0}^{n} \frac{\lambda^{k}(1)_{k+1,1 / \lambda}}{\left(\begin{array}{c}
p+k+1 \\
p+1
\end{array}\right)} S_{2, \lambda}(n, k)\right) \frac{t^{n}}{n !} .
\end{aligned}
$$

Therefore, by comparing the coefficients on both sides of (23), we obtain the following theorem.

Theorem 3 For $n \geq 0$ and $p \geq-1$, we have

$$
\beta_{n, \lambda}^{(p)}=\sum_{k=0}^{n} \frac{\lambda^{k}(1)_{k+1,1 / \lambda}}{\left(\begin{array}{c}
p+k+1 \\
p+1
\end{array}\right)} S_{2, \lambda}(n, k) .
$$

From (6), we get

$$
\begin{aligned}
\sum_{n=k}^{\infty} S_{2, \lambda}(n, k) \frac{t^{n}}{n !} & =\frac{1}{k !}\left(e_{\lambda}(t)-1\right)^{k}=\frac{1}{k !} \sum_{l=0}^{k}\left(\begin{array}{l}
k \\
l
\end{array}\right)(-1)^{k-l} e_{\lambda}^{l}(t) \\
& =\sum_{n=0}^{\infty}\left(\frac{1}{k !} \sum_{l=0}^{k}\left(\begin{array}{l}
k \\
l
\end{array}\right)(-1)^{k-l}(l)_{n, \lambda}\right) \frac{t^{n}}{n !} .
\end{aligned}
$$

By (24), we get

$$
\sum_{l=0}^{k}\left(\begin{array}{l}
k \\
l
\end{array}\right)(-1)^{k-l}(l)_{n, \lambda}= \begin{cases}k ! S_{2, \lambda}(n, k) & \text { if } n \geq k \\
0 & \text { otherwise. }\end{cases}
$$

Let $\triangle$ be a difference operator with $\triangle f(x)=f(x+1)-f(x)$. Then we have

$$
\triangle^{n} f(x)=\sum_{k=0}^{n}\left(\begin{array}{l}
n \\
k
\end{array}\right)(-1)^{n-k} f(x+k)
$$

From (25), we have

$$
k ! S_{2, \lambda}(n, k)=\Delta^{k}(0)_{n, \lambda} \quad(n, k \geq 0, n \geq k) .
$$

In the light of (11), we may consider the degenerate type Eulerian numbers given by

$$
(-1)^{n-m}\left\langle\begin{array}{l}
n \\
m
\end{array}\right\rangle_{\lambda}=\sum_{k=0}^{n-m} \lambda^{k}(1)_{k+1,1 / \lambda}\left(\begin{array}{c}
n-k \\
m
\end{array}\right) \frac{\triangle^{k}(0)_{n, \lambda}}{k !} .
$$

By (26) and (27), we get

$$
(-1)^{n-m}\left\langle\begin{array}{l}
n \\
m
\end{array}\right\rangle_{\lambda}=\sum_{k=0}^{n-m} \lambda^{k}(1)_{k+1,1 / \lambda}\left(\begin{array}{c}
n-k \\
m
\end{array}\right) S_{2, \lambda}(n, k) .
$$


We observe that

$$
\begin{aligned}
\sum_{k=0}^{n} \lambda^{k}(1)_{k+1,1 / \lambda} S_{2, \lambda}(n, k)(t+1)^{n-k} & =\sum_{k=0}^{n} \lambda^{k}(1)_{k+1,1 / \lambda} S_{2, \lambda}(n, k) \sum_{m=0}^{n-k}\left(\begin{array}{c}
n-k \\
m
\end{array}\right) t^{m} \\
& =\sum_{m=0}^{n}\left(\sum_{k=0}^{n-m} \lambda^{k}(1)_{k+1,1 / \lambda} S_{2, \lambda}(n, k)\left(\begin{array}{c}
n-k \\
m
\end{array}\right)\right) t^{m} \\
& =\sum_{m=0}^{n}(-1)^{n-m}\left\langle\begin{array}{l}
n \\
m
\end{array}\right\rangle_{\lambda} t^{m} .
\end{aligned}
$$

From (29) and Theorem 3, we note that

$$
\begin{aligned}
\beta_{n, \lambda}^{(p)} & =\sum_{k=0}^{n} \lambda^{k}(1)_{k+1,1 / \lambda}\left(\begin{array}{c}
p+k+1 \\
k
\end{array}\right)^{-1} S_{2, \lambda}(n, k) \\
& =(p+1) \sum_{k=0}^{n} \lambda^{k}(1)_{k+1,1 / \lambda} S_{2, \lambda}(n, k) \int_{0}^{1} t^{p}(1-t)^{k} d t \\
& =(p+1) \int_{0}^{1} \sum_{k=0}^{n} \lambda^{k}(1-t)^{n} t^{p}(1)_{k+1,1 / \lambda} S_{2, \lambda}(n, k)\left(1+\frac{t}{1-t}\right)^{n-k} d t \\
& =(p+1) \int_{0}^{1}(1-t)^{n} t^{p} \sum_{k=0}^{n}\left\langle\begin{array}{l}
n \\
k
\end{array}\right\rangle_{\lambda}(-1)^{n-k}\left(\frac{t}{1-t}\right)^{k} d t \\
& =(p+1) \sum_{k=0}^{n}\left\langle\begin{array}{l}
n \\
k
\end{array}\right)_{\lambda}(-1)^{n-k} \int_{0}^{1}(1-t)^{n-k} t^{p+k} d t \\
& =(p+1) \sum_{k=0}^{n}\left\langle\begin{array}{l}
n \\
k
\end{array}\right)_{\lambda}(-1)^{n-k} \frac{(n-k) !(p+k) !}{(p+n+1) !} \\
& =\frac{p+1}{n+p+1} \sum_{k=0}^{n}\left\langle\begin{array}{l}
n \\
k
\end{array}\right\rangle_{\lambda}(-1)^{n-k}\left(\begin{array}{l}
p+n \\
p+k
\end{array}\right)^{-1} .
\end{aligned}
$$

Therefore, by (30), we obtain the following theorem.

Theorem 4 For $n, p \geq 0$, we have

$$
\beta_{n, \lambda}^{(p)}=\frac{p+1}{n+p+1} \sum_{k=0}^{n}\left\langle\begin{array}{l}
n \\
k
\end{array}\right\rangle_{\lambda}(-1)^{n-k}\left(\begin{array}{l}
p+n \\
p+k
\end{array}\right)^{-1} .
$$

Let $r$ be a positive integer. The unsigned $r$-Stirling number of the first kind $\left[\begin{array}{l}n \\ k\end{array}\right]_{r}$ is the number of permutations of the set $[n]=\{1,2,3, \ldots, n\}$ with exactly $k$ disjoint cycles in such a way that the numbers $1,2,3, \ldots, r$ are in distinct cycles, while the $r$-Stirling number of the second kind $\left\{\begin{array}{l}n \\ k\end{array}\right\}_{r}$ counts the number of partitions of the set $[n]$ into $k$ non-empty disjoint subsets in such a way that the numbers $1,2,3, \ldots, r$ are in distinct subsets. In [13], Kim et al. introduced the unsigned degenerate $r$-Stirling numbers of the first kind $\left[\begin{array}{l}n \\ k\end{array}\right]_{r, \lambda}$ as a degenerate version of $\left[\begin{array}{l}n \\ k\end{array}\right]_{r}$ and the degenerate $r$-Stirling number of the second kind $\left\{\begin{array}{l}n \\ k\end{array}\right\}_{r, \lambda}$ as a degenerate version of $\left\{\begin{array}{l}n \\ k\end{array}\right\}_{r}$. It is well known that the degenerate $r$-Stirling numbers of 
the second kind are given by

$$
(x+r)_{n, \lambda}=\sum_{k=0}^{n}\left\{\begin{array}{l}
n+r \\
k+r
\end{array}\right\}_{r, \lambda}(x)_{k} \quad(n \geq 1) .
$$

From (31), we note that

$$
\frac{1}{k !}\left(e_{\lambda}(t)-1\right)^{k} e_{\lambda}^{r}(t)=\sum_{n=k}^{\infty}\left\{\begin{array}{l}
n+r \\
k+r
\end{array}\right\}_{r, \lambda} \frac{t^{n}}{n !} \quad(k \geq 0, r \geq 1) .
$$

By the Euler transformation formula in (8) and (32), we get

$$
\begin{aligned}
& \sum_{n=0}^{\infty} \beta_{n, \lambda}^{(p)} \frac{t^{n}}{n !}={ }_{2} F_{1}\left(\begin{array}{c}
1-\lambda, 1 \\
p+2
\end{array} \mid 1-e_{\lambda}(t)\right) \\
& =e_{\lambda}^{p+\lambda}(t) \sum_{k=0}^{\infty} \frac{\langle p+1+\lambda\rangle_{k}\langle p+1\rangle_{k}}{\langle p+2\rangle_{k}} \frac{\left(1-e_{\lambda}(t)\right)^{k}}{k !} \\
& =\frac{p+1}{\langle 1\rangle_{p+1,1 / \lambda}} \sum_{k=0}^{\infty} \frac{\lambda^{k}\langle 1\rangle_{p+k+1,1 / \lambda}}{p+k+1} \frac{(-1)^{k}}{k !}\left(e_{\lambda}(t)-1\right)^{k} e_{\lambda}^{p+\lambda}(t) \\
& =\frac{p+1}{\langle 1\rangle_{p+1,1 / \lambda}} \sum_{k=0}^{\infty} \frac{\lambda^{k}\langle 1\rangle_{p+k+1,1 / \lambda}}{p+k+1}(-1)^{k} \sum_{m=k}^{\infty}\left\{\begin{array}{c}
m+p \\
k+p
\end{array}\right\}_{p, \lambda} \frac{t^{m}}{m !}(1+\lambda t) \\
& =\sum_{m=0}^{\infty} \frac{p+1}{\langle 1\rangle_{p+1,1 / \lambda}} \sum_{k=0}^{m} \frac{\lambda^{k}\langle 1\rangle_{p+k+1,1 / \lambda}}{p+k+1}(-1)^{k}\left\{\begin{array}{l}
m+p \\
k+p
\end{array}\right\}_{p, \lambda} \frac{t^{m}}{m !}(1+\lambda t) \\
& =\sum_{n=0}^{\infty}\left\{\frac{p+1}{\langle 1\rangle_{p+1,1 / \lambda}} \sum_{k=0}^{n} \frac{\lambda^{k}\langle 1\rangle_{p+k+1,1 / \lambda}}{p+k+1}(-1)^{k}\left\{\begin{array}{l}
n+p \\
k+p
\end{array}\right\}_{p, \lambda}\right\} \frac{t^{n}}{n !} \\
& +\sum_{n=1}^{\infty}\left\{\frac{n(p+1)}{\langle 1\rangle_{p+1,1 / \lambda}} \sum_{k=0}^{n-1} \frac{\lambda^{k+1}\langle 1\rangle_{p+k+1,1 / \lambda}}{p+k+1}(-1)^{k}\left\{\begin{array}{c}
n+p-1 \\
k+p
\end{array}\right\}_{p, \lambda}\right\} \frac{t^{n}}{n !}
\end{aligned}
$$

where $\langle x\rangle_{0, \lambda}=1,\langle x\rangle_{n, \lambda}=x(x+\lambda) \cdots(x+(n-1) \lambda)(n \geq 1)$. Therefore, we obtain the following theorem.

Theorem 5 For $n \geq 1$ and $p \geq 0$, we have

$$
\begin{aligned}
\beta_{n, \lambda}^{(p)}= & \frac{p+1}{\langle 1\rangle_{p+1,1 / \lambda}} \sum_{k=0}^{n} \frac{\lambda^{k}\langle 1\rangle_{p+k+1,1 / \lambda}}{p+k+1}(-1)^{k}\left\{\begin{array}{c}
n+p \\
k+p
\end{array}\right\}_{p, \lambda} \\
& +n \lambda \frac{(p+1)}{\langle 1\rangle_{p+1,1 / \lambda}} \sum_{k=0}^{n-1} \frac{\lambda^{k}\langle 1\rangle_{p+k+1,1 / \lambda}}{p+k+1}(-1)^{k}\left\{\begin{array}{c}
n+p-1 \\
k+p
\end{array}\right\}_{p, \lambda} .
\end{aligned}
$$

Note that

$$
\lim _{\lambda \rightarrow 0} \beta_{n, \lambda}^{(p)}=\frac{p+1}{p !} \sum_{k=0}^{n}(-1)^{k} \frac{(p+k) !}{p+k+1}\left\{\begin{array}{l}
n+p \\
k+p
\end{array}\right\}_{p, \lambda} .
$$


From Theorem 3, we have

$$
\begin{aligned}
\sum_{n=0}^{\infty} \beta_{n, \lambda}^{(p)} \frac{t^{n}}{n !} & =\sum_{n=0}^{\infty}\left(\sum_{k=0}^{n} \frac{\lambda^{k}(1)_{k+1,1 / \lambda}}{\left(\begin{array}{c}
p+k+1 \\
p+1
\end{array}\right)} S_{2, \lambda}(n, k)\right) \frac{t^{n}}{n !} \\
& =\sum_{k=0}^{\infty} \frac{\lambda^{k}(1)_{k+1,1 / \lambda}}{\left(^{p+k+1} \begin{array}{c}
p+1 \\
p+1
\end{array}\right)}\left(e_{\lambda}(t)-1\right)^{k} \\
& =(p+1) \sum_{k=0}^{\infty} \frac{p ! k !}{(k+p+1) !} \lambda^{k}(1)_{k+1,1 / \lambda} \frac{1}{k !}\left(e_{\lambda}(t)-1\right)^{k} \\
& =(p+1) \sum_{k=0}^{\infty} \frac{(-1)^{k} \lambda^{k}(1) k+1,1 / \lambda}{k !}\left(1-e_{\lambda}(t)\right)^{k} \int_{0}^{1}(1-x)^{p} x^{k} d x \\
& =(p+1) \sum_{k=0}^{\infty}(-1)^{k}\left(\begin{array}{c}
\lambda-1 \\
k
\end{array}\right)\left(1-e_{\lambda}(t)\right)^{k} \int_{0}^{1}(1-x)^{p} x^{k} d x \\
& =(p+1) \int_{0}^{1}(1-x)^{p}\left(1-x\left(1-e_{\lambda}(t)\right)\right)^{\lambda-1} d x .
\end{aligned}
$$

Therefore, we obtain the following theorem.

Theorem 6 For $p \geq 0$, we have

$$
\sum_{n=0}^{\infty} \beta_{n, \lambda}^{(p)} \frac{t^{n}}{n !}=(p+1) \int_{0}^{1}(1-x)^{p}\left(1-x\left(1-e_{\lambda}(t)\right)\right)^{\lambda-1} d x
$$

\section{Generalized degenerate Bernoulli polynomials}

In this section, we consider the generalized degenerate Bernoulli polynomials which are derived from the Gauss hypergeometric function. In the light of (20), we define the generalized degenerate Bernoulli polynomials by

$$
\sum_{n=0}^{\infty} \beta_{n, \lambda}^{(p)}(x) \frac{t^{n}}{n !}={ }_{2} F_{1}\left(\begin{array}{c}
1-\lambda, 1 \\
p+2
\end{array} \mid 1-e_{\lambda}(t)\right) e_{\lambda}^{x}(t)
$$

When $x=0, \beta_{n, \lambda}^{(p)}(0)=\beta_{n, \lambda}^{(p)}(n \geq 0)$. Thus, by (35), we get

$$
\begin{aligned}
\sum_{n=0}^{\infty} \beta_{n, \lambda}^{(p)}(x) \frac{t^{n}}{n !} & ={ }_{2} F_{1}\left(\begin{array}{c}
1-\lambda, 1 \\
p+2
\end{array} \mid 1-e_{\lambda}(t)\right) e_{\lambda}^{x}(t) \\
& =\sum_{l=0}^{\infty} \beta_{l, \lambda}^{(p)} \frac{t^{l}}{l !} \sum_{m=0}^{\infty}(x)_{m, \lambda} \frac{t^{m}}{m !} \\
& =\sum_{n=0}^{\infty}\left(\sum_{l=0}^{n}\left(\begin{array}{l}
n \\
l
\end{array}\right) \beta_{l, \lambda}^{(p)}(x)_{n-l, \lambda}\right) \frac{t^{n}}{n !} .
\end{aligned}
$$

Therefore, by comparing the coefficients on both sides of (36), we obtain the following theorem. 
Theorem 7 For $n \geq 0$, we have

$$
\beta_{n, \lambda}^{(p)}(x)=\sum_{l=0}^{n}\left(\begin{array}{l}
n \\
l
\end{array}\right) \beta_{l, \lambda}^{(p)}(x)_{n-l, \lambda}
$$

From (35), we note that

$$
\begin{aligned}
\sum_{n=1}^{\infty} \frac{d}{d x} \beta_{n, \lambda}^{(p)}(x) \frac{t^{n}}{n !} & ={ }_{2} F_{1}\left(\begin{array}{c}
1-\lambda, 1 \\
p+2
\end{array} \mid 1-e_{\lambda}(t)\right) \frac{d}{d x} e_{\lambda}^{x}(t) \\
& =\frac{1}{\lambda} \log (1+\lambda t)_{2} F_{1}\left(\begin{array}{c}
1-\lambda, 1 \\
p+2
\end{array} \mid 1-e_{\lambda}(t)\right) e_{\lambda}^{x}(t) \\
& =\frac{1}{\lambda} \sum_{l=1}^{\infty} \frac{(-1)^{l-1} \lambda^{l}}{l} t^{l} \sum_{m=0}^{\infty} \beta_{m, \lambda}^{(p)}(x) \frac{t^{m}}{m !} \\
& =\sum_{n=1}^{\infty}\left(\sum_{l=1}^{n} \frac{(-\lambda)^{l-1}}{l} \frac{n ! \beta_{n-l, \lambda}^{(p)}(x)}{(n-l) !}\right) \frac{t^{n}}{n !}
\end{aligned}
$$

Thus, we have

$$
\frac{d}{d x} \beta_{n, \lambda}^{(p)}(x)=\sum_{l=1}^{n}(-\lambda)^{l-1}(l-1) !\left(\begin{array}{l}
n \\
l
\end{array}\right) \beta_{n-l, \lambda}^{(p)}(x) .
$$

Proposition 8 For $n \geq 1$, we have

$$
\frac{d}{d x} \beta_{n, \lambda}^{(p)}(x)=\sum_{l=1}^{n}(-\lambda)^{l-1}(l-1) !\left(\begin{array}{l}
n \\
l
\end{array}\right) \beta_{n-l, \lambda}^{(p)}(x) .
$$

By (13), we easily get

$$
\begin{aligned}
\sum_{n=k}^{\infty} S_{2, \lambda}(n, k \mid x) \frac{t^{n}}{n !} & =\frac{1}{k !}\left(e_{\lambda}(t)-1\right)^{k} e_{\lambda}^{x}(t) \\
& =\frac{1}{k !} \sum_{l=0}^{k}\left(\begin{array}{l}
k \\
l
\end{array}\right)(-1)^{k-l} e_{\lambda}^{l+x}(t) \\
& =\sum_{n=0}^{\infty}\left(\frac{1}{k !} \sum_{l=0}^{k}(-1)^{k-l}(l+x)_{n, \lambda}\right) \frac{t^{n}}{n !} .
\end{aligned}
$$

Thus we have

$$
\frac{1}{k !} \sum_{l=0}^{k}\left(\begin{array}{l}
k \\
l
\end{array}\right)(-1)^{k-l}(l+x)_{n, \lambda}= \begin{cases}S_{2, \lambda}(n, k \mid x), & \text { if } n \geq k \\
0, & \text { otherwise. }\end{cases}
$$

From (37), we note that

$$
S_{2, \lambda}(n, k \mid x)=\frac{1}{k !} \triangle^{k}(x)_{n, \lambda} \quad(n \geq k) .
$$


Lemma 9 For $n, k \geq 0$ with $n \geq k$, we have

$$
S_{2, \lambda}(n, k \mid x)=\frac{1}{k !} \Delta^{k}(x)_{n, \lambda} \quad(n \geq k) .
$$

Now, we observe that

$$
\begin{aligned}
\sum_{n=0}^{\infty} \beta_{n, \lambda}^{(p)}(x) \frac{t^{n}}{n !} & =\sum_{k=0}^{\infty} \frac{(p+1) ! k !}{(p+k+1) !} \lambda^{k}(1)_{k+1,1 / \lambda} \frac{1}{k !}\left(e_{\lambda}(t)-1\right)^{k} e_{\lambda}^{x}(t) \\
& =\sum_{k=0}^{\infty} \frac{(1)_{k+1,1 / \lambda} \lambda^{k}}{\left(\begin{array}{c}
p+k+1 \\
p+1
\end{array}\right)} \sum_{n=k}^{\infty} S_{2, \lambda}(n, k \mid x) \frac{t^{n}}{n !} \\
& =\sum_{n=0}^{\infty}\left(\sum_{k=0}^{n} \frac{(1)_{k+1,1 / \lambda} \lambda^{k}}{\left(\begin{array}{c}
p+k+1 \\
p+1
\end{array}\right)} S_{2, \lambda}(n, k \mid x)\right) \frac{t^{n}}{n !}
\end{aligned}
$$

Therefore, by (38), we obtain the following theorem.

Theorem 10 For $n \geq 0$, we have

$$
\beta_{n, \lambda}^{(p)}(x)=\sum_{k=0}^{n} \frac{(1)_{k+1,1 / \lambda} \lambda^{k}}{\left(\begin{array}{c}
p+k+1 \\
p+1
\end{array}\right)} S_{2, \lambda}(n, k \mid x)
$$

Remark 11 Let $p$ be a nonnegative integer. Then, by Theorem 7 and (35), we easily get

$$
\begin{aligned}
& \beta_{n, \lambda}^{(p)}(x+y)=\sum_{k=0}^{n}\left(\begin{array}{l}
n \\
k
\end{array}\right) \beta_{k, \lambda}^{(p)}(x)(y)_{n-k, \lambda} \quad(n \geq 0), \\
& \beta_{n, \lambda}^{(p)}(x+1)-\beta_{n, \lambda}^{(p)}(x)=\sum_{k=0}^{n-1}\left(\begin{array}{l}
n \\
k
\end{array}\right) \beta_{k, \lambda}^{(p)}(x)(1)_{n-k, \lambda} \quad(n \geq 1), \\
& \beta_{n, \lambda}^{(p)}(m x)=\sum_{k=0}^{n}\left(\begin{array}{l}
n \\
k
\end{array}\right) \beta_{k, \lambda}^{(p)}(x)(m-1)^{n-k}(x)_{n-k, \lambda / m-1} \quad(n \geq 0, m \geq 2) .
\end{aligned}
$$

\section{Conclusion}

This work was motivated by Rahmani's paper [14] in which a new family of $p$-Bernoulli numbers and polynomials was constructed by means of the Gauss hypergeometric function. This family of numbers and polynomials generalizes the classical Bernoulli numbers and polynomials, in the sense that they reduce to the classical Bernoulli numbers and polynomials for $p=0$. In the light of the regained recent interests in them, we were interested in finding a degenerate version of those numbers and polynomials. Indeed, the generalized degenerate Bernoulli numbers and polynomials, which reduce to the Carlitz degenerate Bernoulli numbers and polynomials for $p=0$, were constucted in terms of the Gauss hypergeometric function. Moreover, the degenerate type Eulerian numbers were introduced as a degenerate version of Eulerian numbers.

In this paper, we expressed the generalized degenerate Bernoulli numbers in terms of the degenerate Stirling numbers of the second kind, of the degenerate type Eulerian numbers, of the degenerate $p$-Stirling numbers of the second kind and of an integral on the unit 
interval. In addition, we represented the generalized degenerate Bernoulli polynomials in terms of the degenerate Stirling polynomials of the second kind.

It is one of our future projects to continue pursuing this line of research. Namely, by studying degenerate versions of some special polynomials and numbers, we want to find their applications in mathematics, science and engineering.

\section{Acknowledgements}

The authors would like to thank the reviewers for their valuable comments and suggestions and Jangjeon Institute for Mathematical Science for the support of this research.

\section{Funding}

The first author has been conducted by the Research Grant of Kwangwoon University in 2021.

\section{Availability of data and materials}

Not applicable.

\section{Competing interests}

The authors declare that they have no competing interests.

\section{Authors' contributions}

TK and DSK conceived of the framework and structured the whole paper; DSK and TK wrote the paper; HL typed; LCJ, and HYK checked the results of the paper; DSK and TK completed the revision of the paper. All authors have read and approved the final version of the manuscript.

\section{Author details}

'Department of Mathematics, Kwangwoon University, Seoul, 139-701, Republic of Korea. ${ }^{2}$ Department of Mathematics, Sogang University, Seoul, 121-742, Republic of Korea. ${ }^{3}$ Graduate School of Education, Konkuk University, Seoul, 143-701, Republic of Korea.

\section{Publisher's Note}

Springer Nature remains neutral with regard to jurisdictional claims in published maps and institutional affiliations.

Received: 4 January 2021 Accepted: 9 March 2021 Published online: 19 March 2021

\section{References}

1. Andrews, G.E., Askey, R., Roy, R.: Special Functions. Cambridge University Press, Cambridge (1999)

2. Bailey, W.N.: Generalized Hypergeometric Series. Cambridge Tracts in Mathematics and Mathematical Physics, vol. 32. Stechert-Hafner, New York (1964)

3. Carlitz, L:: Degenerate Stirling, Bernoulli and Eulerian numbers. Util. Math. 15, 51-88 (1979)

4. Comtet, L.: Advanced Combinatorics. Reidel, Dordrecht (1974)

5. Graham, R.L., Knuth, D.E., Patashnik, O.: Concrete Mathematics: A Foundation for Computer Science, 2nd edn. Addison-Wesley, Reading (1994)

6. Jang, L.-C., Kim, D.S., Kim, T., Lee, H.: Some identities involving derangement polynomials and numbers and moments of gamma random variables. J. Funct. Spaces 2020, Article ID 6624006 (2020)

7. Kim, D.S., Kim, T.: A note on a new type of degenerate Bernoulli numbers. Russ. J. Math. Phys. 27(2), $227-235$ (2020)

8. Kim, T.: A note on degenerate Stirling polynomials of the second kind. Proc. Jangjeon Math. Soc. 20(3), 319-331 (2017)

9. Kim, T., Kim, D.S.: Note on the degenerate gamma function. Russ. J. Math. Phys. 27(3), 352-358 (2020)

10. Kim, T., Kim, D.S., Kim, H.Y., Kwon, J.: Degenerate Stirling polynomials of the second kind and some applications. Symmetry 11(8), $1046(2019)$

11. Kim, T., Kim, D.S., Kim, H.Y., Kwon, J.: Erratum Kim, T. et al. Degenerate Stirling polynomials of the second kind and some applications. Symmetry 11(8), 1046 (2019). Symmetry 11(12), 1530 (2019)

12. Kim, T., Kim, D.S., Lee, H., Kwon, J.: Degenerate binomial coefficients and degenerate hypergeometric functions. Adv. Differ. Equ. 2020, Article ID 115 (2020)

13. Kim, T., Kim, D.S., Lee, H., Park, J.-W.: A note on degenerate $r$-Stirling numbers. J. Inequal. Appl. 2020, Article ID 225 (2020)

14. Rahmani, M.: On p-Bernoulli numbers and polynomials. J. Number Theory 157, 350-366 (2015) 